\title{
Prevalência de fissura de palato submucosa associada à fissura labial
}

\section{Prevalence of submucous cleft palate associated with cleft lip}

\author{
Camila Queiroz de Moraes Silveira Di Ninno', Karina Cristiane Gonçalves², Mariana Santos Braga², \\ Izabel Cristina Campolina Miranda ${ }^{2}$
}

\begin{abstract}
RESUMO
Objetivo: Investigar a prevalência de fissura de palato submucosa associada à fissura labial, sua correlação com o gênero, tipo de fissura labial, sinais e sintomas apresentados. Métodos: Estudo baseado na coleta de dados de 132 pacientes com fissura labial consecutivos de um centro especializado em fissura labiopalatina, de ambos os gêneros, coletados pela mesma fonoaudióloga na primeira consulta do paciente no centro. Foram realizados palpação do palato duro, avaliação visual do palato mole e úvula e questionário aos pacientes ou responsáveis sobre a presença de sintomas associados. Resultados: Da amostra total investigada, a maioria dos pacientes era do gênero masculino e apresentava fissura de lábio unilateral esquerda. Onze por cento dos casos apresentavam sinais de fissura submucosa. Dentre eles, a ocorrência de refluxo nasal foi encontrada em alguns casos (21\%), histórico de otite em metade dos casos e nenhum deles apresentava fala com sinais de hipernasalidade. Conclusão: Onze por cento dos pacientes com fissura labial apresentam sinais de fissura de palato submucosa, sendo sua prevalência maior no gênero masculino e na fissura labial do tipo unilateral esquerda.
\end{abstract}

Descritores: Fenda labial; Fissura palatina/diagnóstico; Fissura palatina/patologia; Fissura palatina/fisiopatologia; Anormalidades congênitas; Sinais e sintomas

\section{INTRODUÇÃO}

A fissura de palato submucosa (FPSM) é um tipo de malformação congênita que pode apresentar-se de forma isolada, associada à fissura labial ou associada a síndromes ${ }^{(1,2)}$.

A prevalência da FPSM varia de 1/1200 a 1/2500 nascidos e independe de fatores étnico-geográficos. No entanto, alguns autores ressaltam que diferenças nos valores de prevalência da FPSM na população podem ser atribuídas a características da amostra selecionada e critérios utilizados em cada estudo para o diagnóstico da FPSM ${ }^{(3-5)}$.

As fissuras labiais com ou sem envolvimento do palato, apresentam prevalência de $1 / 600$ recém nascidos ${ }^{(6,7)}$. Enquanto estudos verificaram prevalência de $12 \%$ e $13 \%$ de FPSM em casos com fissura labial $(\mathrm{FL})^{(2,8)}$, verificou-se em investigação de pacientes com FPSM, que dos 75 indivíduos com FPSM

Pesquisa realizada no Curso de Fonoaudiologia, Pontifícia Universidade Católica de Minas Gerais - PUC Minas - Belo Horizonte (MG), Brasil.

(1) Curso de Fonoaudiologia da Pontifícia Universidade Católica de Minas Gerais - PUC Minas - Belo Horizonte (MG), Brasil. Centro de Tratamento e Reabilitação de Fissuras Labiopalatinas e Deformidades Craniofaciais - PUC Minas/Hospital da Baleia - Belo Horizonte (MG), Brasil.

(2) Curso de Fonoaudiologia da Pontifícia Universidade Católica de Minas Gerais - PUC Minas - Belo Horizonte (MG), Brasil.

Endereço para correspondência: Camila Queiroz de Moraes Silveira Di Ninno. Av. Dom José Gaspar, 500, Prédio 25, Coração Eucarístico, Belo Horizonte (MG), Brasil, CEP: 30535-610. E-mail: camilaninno@uol.com.br Recebido em: 28/7/2010; Aceito em: 21/11/2010 analisados, $25 \%$ tinham associação com a fissura labial (FL) ${ }^{(9)}$.

Em relação ao diagnóstico da FPSM, este é feito por meio de inspeção intra-oral para a busca de sinais clássicos que identificam a FPSM: úvula bífida, diástase da musculatura velar na linha média e chanfradura óssea na borda posterior do palato duro ${ }^{(8,10)}$. Entretanto, estas características podem apresentar-se de forma associada ou não, sendo indispensável para o diagnóstico da FPSM, a presença de diástase muscular no palato mole $\mathrm{e}^{(11)}$.

Apesar de haver sinais característicos da FPSM, a dificuldade de sua identificação logo ao nascimento leva muitas vezes ao diagnóstico tardio, em geral realizado quando há o aparecimento de sintomas evidentes de disfunção velofaríngea $(\mathrm{DVF})^{(12)}$. No entanto, é sabido que este fato pode ser evitado e que condutas adequadas podem ser adotadas, quando o diagnóstico precoce é realizado. Por essas razões, torna-se essencial a divulgação dos sinais e sintomas da FPSM a fonoaudiólogos, médicos e dentistas, para facilitação do diagnóstico precoce. Assim, é possível o acompanhamento das doenças da orelha média, das alterações da fala decorrentes da DVF e do cuidado na indicação de adenoidectomia. Casos assintomáticos de FPSM, muitas vezes tornam-se sintomáticos após a realização de adenoidectomia, por levar a uma DVF, o que pode ser desfavorável ao indivíduo ${ }^{(2,12-15)}$. Portanto, a indicação nestes casos deve levar em conta critérios específicos ${ }^{(11,12,16)}$.

Embora a grande maioria dos casos de FPSM seja assintomática, o fonoaudiólogo deve acompanhar os bebês com 
diagnóstico de FPSM até que seja possível avaliar a presença de DVF e, caso necessário, intervir a tempo, definindo a conduta necessária. Para os casos sintomáticos, aponta-se a alteração anatômica como fator causal para a DVF, a qual pode vir a gerar dificuldades alimentares, auditivas e de fala $a^{(2,5,10,12,17,18)}$.

Dentre as alterações alimentares estão a presença de refluxo nasal de alimentos durante a deglutição e o tempo de mamada prolongada acima de 40 minutos, devido à fraca pressão intraoral negativa durante a sucção ${ }^{(15,19)}$.

Com relação à audição, há uma predisposição a otites médias crônicas, que trazem o risco de perdas auditivas, principalmente do tipo condutiva. Estas alterações auditivas, comuns em indivíduos com fissura de palato, estão associadas ao mau funcionamento da tuba auditiva ${ }^{(2,12,15,18,20)}$.

A ineficiência do fechamento tubário deve-se à deficiência do músculo tensor do véu palatino. A alteração condutiva pode prejudicar tanto a acuidade, quanto a percepção auditiva, e é um dos fatores predisponentes a alterações na fala ${ }^{(16,21)}$.

A fala na FPSM sintomática é caracterizada por hipernasalidade $^{(12)}$, manifestação mais frequente da DVF na fala, associada ao escape de ar nasal e à fraca pressão intra-oral em plosivos e fricativos ${ }^{(14,22)}$.

Para os casos sintomáticos, existe a necessidade de uma correção cirúrgica (palatoplastia) das estruturas palatinas, fonoterapia e/ou utilização de prótese de palato $^{(12,17,23)}$.

Para os casos com indicação cirúrgica, estudos demonstraram resultados eficazes em relação à fala, ressonância e função velofaríngea (FVF), quando realizada intervenção precoce em indivíduos com FPSM $^{(9)}$.

A fonoterapia é indicada em casos que apresentam distúrbios compensatórios como golpes de glote e fricativas faríngeas. Em estudos realizados com o objetivo de levantar a ocorrência de indicação de cirurgia de palato na população atendida no Hospital de Reabilitação de Anomalias Craniofaciais da Universidade de São Paulo (HRAC-USP), dos 1.267 casos com diagnóstico de FPSM, 49\% eram sintomáticos, e destes, em $57 \%$ a conduta indicada foi a fonoterapia, visando a melhora da inteligibilidade de fala. Neste mesmo estudo, entre os casos que foram indicados para adaptação de prótese de palato (2\%), metade relacionava-se a quadros sindrômicos, sendo a Síndrome Velocardiofacial a mais frequente ${ }^{(17)}$.

Como não existem muitos estudos acerca da incidência de FPSM associada à fissura labial e pelo fato de, na prática clínica, ser observada ocorrência desta associação de forma considerável, acredita-se que a definição da prevalência desta condição poderá alertar outros profissionais da saúde a realizarem um exame intra-oral minucioso em todos os casos com FL. Assim, estes casos poderão ser acompanhados e a definição de conduta estabelecida o mais precocemente possível, evitando intervenções tardias e prognóstico desfavorável ao indivíduo com FPSM.

Por saber que a FPSM apresenta prevalência de 0,4\% a $0,83 \%$ nascimentos, que a úvula bífida, importante marcador para FPSM tem prevalência de 1,25\% entre caucasóides e $0,4 \%$ entre negróides e que acometem $0,16 \%$ dos nascidos, torna-se então plausível a verificação da prevalência, também de FPSM associada à FL, para que se torne rotineira a inspeção intra-oral em indivíduos que aparentemente apresentam somente $\mathrm{FL}^{(6,7)}$.
De acordo com nossa experiência clínica e relatos da literatura, espera-se encontrar entre os casos de FL, por volta de $10 \%$ com FPSM associada, número este que consideramos expressivo, tendo em vista que, em geral, não são diagnosticados precocemente ${ }^{(2)}$.

Acredita-se que neste estudo será encontrada maior ocorrência de FPSM em indivíduos do gênero masculino, embora a FPSM possa ser considerada um subtipo de fissura de palato e, portanto, mais frequente no gênero feminino. Nosso argumento para sustentar essa hipótese é que o estudo abordará apenas casos com FL, que tem maior ocorrência no gênero masculino (2:1).

Os objetivos deste estudo foram identificar a prevalência de fissura submucosa em pacientes com fissura labial isolada, recebidos para triagem no Centro de Tratamento e Reabilitação de Fissuras Labiopalatais e Deformidades Craniofaciais (Centrare), analisar se há relação entre o gênero e a prevalência de FPSM, pesquisar se há associação entre FPSM e o tipo de fissura labial, identificar os sinais mais frequentes apresentados nos pacientes com FPSM e verificar se há sintomas da DVF nos casos com FPSM.

\section{MÉTODOS}

O presente estudo foi aprovado pelo Comitê de Ética em Pesquisa da Pontifícia Universidade Católica de Minas Gerais e do Hospital Baleia (CAAE 0092.0.213.000-08).

Neste estudo foram analisados os dados de todos os pacientes do Centro de Tratamento e Reabilitação de Fissuras Labiopalatais e Deformidades Craniofaciais (Centrare - PUC Minas/ Hospital da Baleia), atendidos entre os meses de fevereiro de 2008 e março de 2009. Foram incluídos todos os pacientes que apresentavam diagnóstico de fissura labial do tipo pré-forame incisivo uni ou bilateral, segundo a classificação de Spina ${ }^{(24)}$, independente de idade e gênero. Como o objetivo era investigar a presença de fissura de palato submucosa, foram excluídos os casos que apresentavam fissura de palato aberta, mesmo que incompleta, e também os casos sindrômicos, com alterações neurológicas ou quaisquer outras más formações craniofaciais associadas.

Todos os incluídos na pesquisa haviam sido avaliados pela mesma fonoaudióloga, uma das pesquisadoras deste estudo, que rotineiramente realiza a inspeção clínica intra-oral em todos os pacientes com fissura labial. Esta avaliação abrange a palpação do palato, para identificação de chanfradura óssea, e a visualização do palato mole e úvula durante a emissão da vogal /a/ ou do reflexo de vômito, para investigação da presença de diástase muscular e úvula bífida ou sulcada, sinais da presença de FPSM.

Foram também analisados os dados coletados com os pais ou acompanhantes, no caso de bebês e crianças, e com os próprios pacientes adolescentes e adultos sobre a presença de sintomas que poderiam ser decorrentes de DVF, como a dificuldade para manter o aleitamento no seio materno, a presença de refluxo nasal de alimentos durante a deglutição, o histórico de otite média, e a fala com hipernasalidade e distúrbio articulatório compensatório.

Os dados coletados foram registrados em protocolo de- 
senvolvido especificamente para este estudo (Anexo 1), contendo as iniciais do nome dos pacientes, idade, gênero, sinais característicos de FPSM, tais como: úvula bífida, sulcada ou hipoplásica, diástase muscular e chanfradura óssea; e sintomas de DVF, como sucção, refluxo nasal, otites médias e fala, nos pacientes acima de 18 meses.

O cálculo da prevalência foi realizado a partir da proporção de casos com sinais de fissura de palato submucosa encontrados na população de pacientes do Centro com fissura labial, atendidos no período estudado. As variáveis investigadas foram analisadas por meio do teste Qui-quadrado de Pearson $\left(x^{2}\right)$ e teste Qui-quadrado exato, ambos com nível de significância de $5 \%$.

\section{RESULTADOS}

No período de fevereiro de 2008 a março de 2009, foram atendidos no Centrare 132 pacientes com diagnóstico de fissura pré-forame incisivo. Destes pacientes, $14(11 \%)$ foram diagnosticados como apresentando FPSM associada à fissura labial (Figura 1), ou seja, para cada 100 pacientes com fissura labial, encontrou-se uma prevalência de 11 casos com fissura de palato submucosa associada.

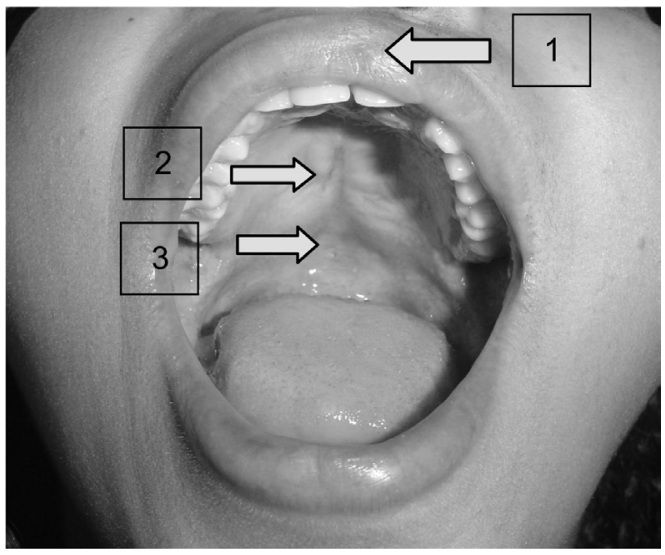

Figura 1. Fissura pré-forame unilateral esquerda operada (1), associada à fissura de palato submucosa com dois sinais clínicos: chanfradura óssea (2) e diástase muscular (3)

As variáveis analisadas: gênero e idade do paciente, o tipo de fissura pré-forame, a existência de alteração no palato duro e/ou mole e a presença de sintomas como refluxo nasal de alimentos, otites, alterações de fala e tempo de aleitamento no seio materno, serão descritas a seguir.
Em relação ao gênero, do total de pacientes analisados, $69(52 \%)$ eram do gênero masculino e $63(48 \%)$ do feminino. Dos 69 pacientes do gênero masculino, dez (14\%) foram diagnosticados com FPSM associada à FL. Das 63 pacientes do gênero feminino, quatro (6\%) apresentaram associação com a FPSM (Tabela 1). Ao se considerar apenas os 14 pacientes com FPSM associada à FL, dez (71\%) eram do gênero masculino e quatro (29\%) do gênero feminino (Tabela 1).

Tabela 1. Distribuição dos pacientes com fissura labial segundo o gênero e o grupo

\begin{tabular}{lccc}
\hline & \multicolumn{2}{c}{ Gênero } & Total \\
\cline { 2 - 3 } Tipo de fissura (Grupo) & $\begin{array}{c}\text { Feminino } \\
\mathrm{n}(\%)\end{array}$ & $\begin{array}{c}\text { Masculino } \\
\mathrm{n}(\%)\end{array}$ & $\mathrm{n}(\%)$ \\
\hline $\begin{array}{l}\text { Fissura labial com submucosa } \\
\text { (FPSM) }\end{array}$ & $4(6,4)$ & $10(14,5)$ & $14(10,6)$ \\
$\begin{array}{l}\text { Fissura labial sem submucosa } \\
\text { (FP) }\end{array}$ & $59(93,7)$ & $59(85,5)$ & $118(89,4)$ \\
Total & 63 & 69 & 132 \\
\hline \multicolumn{3}{c}{$\mathrm{X}^{2}=2,30 ; \mathrm{p}=0,129$} \\
\hline
\end{tabular}

Teste Qui-quadrado $(p \leq 0,05)$

O teste aplicado não revelou resultado significativo quanto à prevalência de um dos gêneros sobre os grupos de indivíduos com e sem a associação da FPSM e FL.

No momento da avaliação, os pacientes apresentavam idade entre 28 dias e 38 anos, com média de oito anos (DP=7 anos). Quando analisados apenas os pacientes com FPSM, a média e o desvio padrão se mantiveram os mesmos (Tabela 2).

Tabela 2. Caracterização da idade dos indivíduos portadores de fissura de palato submucosa

\begin{tabular}{lcc}
\hline Dados & Dias & Anos \\
\hline Mínimo de dias & 115 & 0 \\
Máximo de dias & 6201 & 19 \\
Média de dias & 2857 & 8 \\
DP & 2214 & 7 \\
Mediana & 2833 & 6,5 \\
\hline
\end{tabular}

Legenda: DP = desvio-padrão

Em relação ao tipo de fissura pré-forame, na amostra total analisada, a fissura pré-forame incisivo do tipo unilateral à esquerda e incompleta foi a mais prevalente (Tabela 3). Dos 14 casos com FPSM, 11 pacientes (79\%) apresentavam fis-

Tabela 3. Distribuição dos pacientes com fissura labial segundo o grupo e a subclassificação da fissura

\begin{tabular}{llcc}
\hline Tipo de fissura & Subclassificação & $\mathrm{n}(\%)$ & Qui-quadrado \\
\hline & Uni-lateral esquerda & $10(71,4)$ & $\mathrm{x}^{2}=9,57$ \\
Fissura labial com submucosa (FPSM) & Uni-lateral direita & $1(7,1)$ & $\mathrm{p}=0,008^{\star}$ \\
& Bilateral & $3(21,4)$ & $70(59,3)$ \\
Fissura labial sem submucosa (FP) & Uni-lateral esquerda & $36(30,5)$ & $\mathrm{x}^{2}=43,19$ \\
& Uni-lateral direita & $12(10,2)$ & $\mathrm{p}<0,0005$ \\
\hline
\end{tabular}

\footnotetext{
* Restrição do teste: Todas as células têm valores esperados menores do que 5
}

Teste Qui-quadrado exato $\left(X^{2}=9,57 ; p=0,006\right)$ 
sura pré-forame do tipo unilateral, sendo 10 à esquerda e 1 à direita e $3(21 \%)$ apresentavam fissura pré-forame do tipo bilateral (Tabela 3 ).

Os testes aplicados mostraram resultados significativos quanto à subclassificação das fissuras, no grupo de indivíduos com associação das FPSM e FL, o que justifica assumir que há distribuição heterogênea neste grupo, assim como entre os indivíduos com FL isolada.

Dos 14 pacientes com sinais de FPSM, 11 deles (79\%), apresentavam chanfradura óssea na borda posterior do palato duro e 13 (93\%) apresentavam alterações no palato mole, sendo 11 com diástase muscular, nove com úvula bífida, dois com úvula sulcada e um com a úvula alargada (Figura 2). Entre esses, foi possível observar a presença da tríade clássica em oito indivíduos $(57 \%)$.

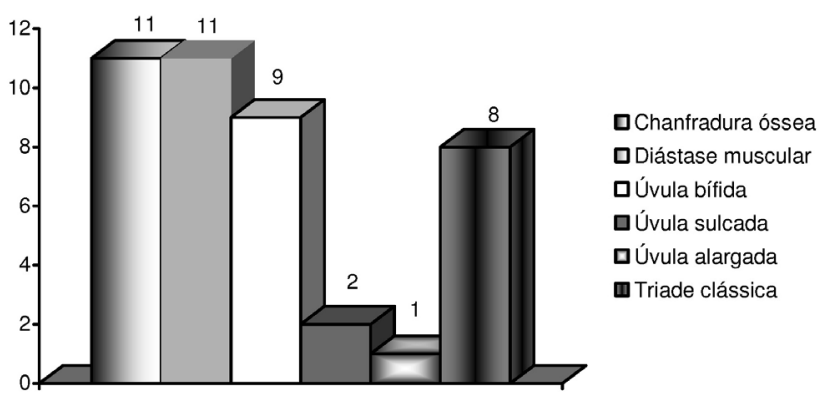

Figura 2. Caracterização dos sinais presentes nos indivíduos com fissura de palato submucosa

Quanto à presença de sintomas que poderiam ser decorrentes da DVF, dos 14 pacientes com FPSM, três (21\%) relataram a ocorrência de refluxo nasal de alimentos durante a deglutição e sete $(50 \%)$ apresentaram histórico de otite média. Não houve nenhum caso com alterações de fala como hipernasalidade ou distúrbio articulatório compensatório.

Sobre a amamentação no seio materno, do total de pacientes investigados, 19 (14\%) não foram amamentados no seio materno e 44 (33\%) foram amamentados durante pelo menos seis meses de idade. Dos pacientes com FPSM, três (21\%) não foram amamentados e apenas outros três (21\%) foram amamentados até pelo menos seis meses.

\section{DISCUSSÃO}

No presente estudo encontrou-se prevalência de $11 \%$ de fissura de FPSM em pacientes com diagnóstico de fissura préforame incisivo. Esta prevalência é semelhante a estudos anteriores, de indivíduos com as duas malformações associadas ${ }^{(2,8)}$.

Quanto ao gênero, tanto em relação à amostra total de indivíduos deste trabalho, quanto ao grupo de pacientes diagnosticados com FPSM, houve predomínio de pacientes do gênero masculino. Outros autores também obtiveram resultados similares em pacientes com FPSM ${ }^{(12,25,26)}$. Sabendo-se que a fissura palatina é mais frequente no gênero feminino e que a FPSM é um subtipo de fissura palatina, poder-se-ia esperar maior prevalência do gênero feminino. Nosso achado de prevalência no gênero masculino pode ser justificado pelo fato de que nossa análise se limitou aos pacientes portadores de fissura labial que, de acordo com a literatura, é mais prevalente no gênero masculino ${ }^{(12,25,26)}$.

$\mathrm{O}$ fato de os pacientes com FPSM, neste trabalho, terem sido diagnosticados em média aos oito anos de idade, confirma que este diagnóstico ainda tem sido tardio para muitos pacientes $^{(12)}$. Tal fato pode ser justificado por estes pacientes serem, até o momento, assintomáticos em relação à DVF. De acordo com outros pesquisadores, a identificação precoce da FPSM é importante para que o paciente e sua família recebam orientações e sejam monitorados quanto à presença de sinais e sintomas de $\mathrm{DVF}^{(12,26-28)}$. Há necessidade da disseminação do conhecimento sobre os sinais da FPSM, entre acadêmicos e profissionais da área da saúde para propiciar sua detecção precoce e o acompanhamento de tais casos.

Nesta pesquisa, a ausência de sintomas característicos de DVF em pacientes com diagnóstico de FPSM, contrapõe-se a outros estudos da literatura, cujos valores oscilam entre $20 \%$ e $98 \%$ de casos de FPSM sintomáticos, cuja manifestação mais evidente é a hipernasalidade ${ }^{(12,14,27,29)}$. A diferença entre o presente estudo e os demais é que os pacientes procuraram o serviço especializado devido à presença da fissura labial. O mesmo não ocorre nos casos em que a FPSM se manifesta de forma isolada, em que os pacientes só procuram o serviço quando apresentam sintomas de DVF, como a hipernasalidade. O estudo que mostra $98 \%$ de casos sintomáticos foi realizado a partir de casos em fonoterapia ${ }^{(29)}$. Deve-se ressaltar que a forma utilizada para a identificação da hipernasalidade foi a avaliação perceptiva. Estudo comparando as avaliações clínicas e instrumentais em pacientes com fissura de palato submucosa assintomática ressaltou a importância do uso combinado de métodos perceptivos e instrumentais e o acompanhamento criterioso e periódico dos casos nos quais a avaliação instrumental mostra sinais de DVF, independente da avaliação clínica $^{(28)}$. Vale ainda lembrar que devido à idade, alguns dos pacientes do presente estudo não haviam desenvolvido a fala no momento da coleta dos dados, o que não permitiu a avaliação da ressonância oronasal.

De acordo com a literatura ${ }^{(10,19)}$, entre as alterações alimentares relacionadas à DVF, em casos com FPSM, pode-se encontrar o refluxo nasal de alimentos durante a deglutição, manifestação encontrada em $21 \%$ dos pacientes com FPSM analisados nesta pesquisa.

No que diz respeito à presença de otite média, metade dos pesquisados relataram este sintoma. Esta informação corrobora a literatura que relaciona a ocorrência de otites médias ao mau funcionamento da tuba auditiva e à deficiência do músculo tensor do véu palatino. A FPSM é apontada como indicador de otites médias agudas graves e de efusão recorrentes, o que torna a intervenção clínica indispensável à saúde auditiva do indivíduo $^{(2,3,18,21)}$.

Quanto à amamentação no seio materno, uma pequena parcela dos pacientes com FPSM associadas à FL, foi amamentada até pelo menos seis meses de idade no seio materno. A justificativa mais frequente para a amamentação com duração inferior a seis meses foi a retirada do seio materno após a primeira intervenção cirúrgica, a queiloplastia. Em um estudo recente, metade das crianças diagnosticadas com algum tipo de fissura tinham em sua dieta a presença do leite materno ${ }^{(25)}$. 
Visto que ainda há falta de conhecimento familiar sobre a primeira opção de alimentação, o leite materno, os resultados encontrados denotam a importância da divulgação dos benefícios decorrentes do aleitamento materno, assim como a necessidade de orientações individualizadas às famílias, quanto a posturas e facilitações adotadas para ingestão satisfatória do alimento em bebês fissurados ${ }^{(25,30)}$.

Observou-se que a tríade clássica ${ }^{(11)}$ da FPSM foi encontrada na maioria dos pacientes, resultado esse similar ao encontrado em outros estudos ${ }^{(27,28)}$.

$\mathrm{Na}$ presente investigação, o tipo de fissura labial mais prevalente foi a fissura pré-forame unilateral à esquerda incompleta, o que comprova os achados de outros autores ${ }^{(24,26)}$.

Tendo em vista os resultados obtidos neste estudo, observase a necessidade da divulgação do conhecimento sobre os sinais e sintomas presentes na FPSM aos profissionais da área da saúde, a fim de propiciar o diagnóstico precoce, acompanhamento e orientações familiares e a intervenção cirúrgica a tempo, quando necessária.

\section{CONCLUSÃO}

A partir deste estudo obteve-se uma prevalência de 11\% de FPSM em pacientes com fissura labial, sendo esta prevalência maior no gênero masculino e na fissura labial do tipo unilateral esquerda.

A maioria dos casos apresentava a tríade clássica da FPSM, caracterizada pela presença de chanfradura óssea, diástase muscular e úvula bífida e nenhum dos pacientes com FPSM deste estudo apresentava hipernasalidade.

\begin{abstract}
Purpose: To investigate the prevalence of submucous cleft palate associated with cleft lip, and its correlation with gender, type of cleft lip, signs and symptoms. Methods: Study based on the data of 132 consecutive patients with cleft lip at a specialized cleft lip and palate center, of both genders, collected by the same speech-language pathologist during the patients' first consultation at the center. Palpation of the hard palate, visual assessment of the soft palate and uvula, and an interview with patients or their parents regarding the presence of associated symptoms were performed. Results: From the total sample, most patients were male and had left-sided cleft lip. Eleven percent of the cases presented signs of submucous cleft palate. Among them, nasal reflux was found in some cases $(21 \%)$, history of otitis in half of the subjects, and none of them presented speech with hypernasality signs. Conclusion: Eleven percent of the patients with cleft lip presented signs of submucous cleft palate, with higher prevalence in males and in left-sided cleft lip.
\end{abstract}

Keywords: Cleft lip; Cleft palate/diagnosis; Cleft palate/pathology; Cleft palate/physiopathology; Congenital abnormalities; Signs and symptoms

\title{
REFERÊNCIAS
}

1. Minami trt, kaplan EN, Wu G, Jobe RP. Velopharyngeal incompetence without overt cleft palate. A collective review and experience with 98 patients. Plast Reconstr Surg. 1975;55(5):573-87.

2. Kono D, Young L, Holtmann B. The association of submucous cleft palate and clefting of the primary palate. Cleft Palate J. 1981;18(3):2079.

3. Weatherley-White RC, Sakura CY Jr, Brenner LD, Stewart JM, Ott JE. Submucous cleft palate. Its incidence, natural history, and indications for treatment. Plast Reconstr Surg. 1972;49(3):297-304.

4. McWilliams BJ. Submucous clefts of the palate: how likely are they to be symptomatic? Cleft Palate Craniofac J. 1991;28(3):247-9

5. Chen KT, Wu J, Noordhoff SM. Submucous cleft palate. Changgeng Yi Xue Za Zhi. 1994;17(2):131-7.

6. Mossey PA, Little J. Epidemiology of oral cleft: an international perspective. In: Wyszynski DF. Cleft Lip and Palate from origin to treatment. New York: Oxford University Press; 2002. p.127-58.

7. World Helth Organization - WHO. Global strategies to reduce the health care burden of craniofacial anomalies. Geneva: WHO; 2002.

8. Gosain AK, Conley SF, Santoro TD, Denny AD. A prospective evaluation of the prevalence of submucous cleft palate in patients with isolated cleft lip versus controls. Plast Reconstr Surg. 1999;103(7):1857-63.

9. Ninno CQ, Genaro KF. Fissura de palato submucosa. Pró-Fono. 1998;10(2):28-33.

10. Calnan J. Submucous cleft palate. Br J Plast Surg. 1954;6(4):264-82.

11. Park S, Omori M, Kato K, Nitta N, Kitano I, Masuda T. Cephalometric analysis in submucous cleft palate: comparison of cephalometric data obtained from submucous cleft palate patients with velopharyngeal competence and incompetence. Cleft Palate Craniofac J. 2002;39(1):105-9.

12. Reiter R, Haase S, Brosch S. [Submucous cleft palate--an often late diagnosed malformation]. Laryngorhinootologie. 2010;89(1):29-33. German.

13. Jesus MS, Penido FA, Valente P. Avaliações fonoaudiológicas clínica e instrumental em indivíduos com fissura labiopalatina. In: Jesus MS, Di Ninno CQ. Fissura labiopalatina: fundamentos para a prática fonoaudiológica. São Paulo: Roca; 2009. p. 57-75.

14. Abyholm FE. Submucous cleft palate. Scand J Plast Reconstr Surg.1976;10(3):209-12.

15. Moss AL, Piggot RW, Jones KJ. Submucous cleft palate: a treatable condition that is commonly missed. Br Med J. 1998;297(5):85-6.

16. Bowen DG, Lehman Junior JA, Neiman GS. Management of submucous cleft palate. In: Transactions $8^{\text {th }}$ International Congress on Cleft Palate and Relates Craniofacial Anomalies. Singapore; 1997. p. 789-93.

17. Betoni VC. Fissura de palato submucosa e realizações de palatoplastia; estudo retrospectivo do HRAC/USP [monografia]. Bauru: Universidade de São Paulo; 2006.

18. Berera G. Index of suspicious. Case 1. Submucous cleft palate. Pediatr Rev. 1993;14(5):191-2.

19. Conley SF, Gosain AK, Marks SM, Larson DL. Identification and assessment of velopharyngeal inadequacy. Am J Otolaryngol. 1997;18(1):38-46.

20. Brandão GR, Genaro KF. Fissura de palato submucosa e audição. Acta Awho. 1998;17:97-104. 
21. Bluestone CD, Shurin PA. Middle ear disease in children: pathogenesis, diagnosis and management. Pediatr Clin North Am. 1974;21(2):379400.

22. Pegoraro-Krook MI, Genaro KF. Communicative disorders in craniofacial malformations. Braz J Dysmorphol Speech Dis. 1981;1(1):35-40.

23. Bzoch KR. Communicative disorders related to cleft lip and palate. Lake Elsinore (CA): Pro-Ed; 1997. Introduction to the study of communicative disorders in cleft palate craniofacial anomalies; p.1-44.

24. Spina V. A proposed modification for the classification of cleft lip and cleft palate. Cleft Palate J. 1973;10:251-2.

25. Rocha CM. Resultados da intervenção interdisciplinar precoce em crianças com fissura labiopalatal atendidas no centro de tratamento de fissuras [dissertação]. Belo Horizonte: Universidade Federal de Minas Gerais, Faculdade de Medicina; 2008.

26. França CM. Incidência das fissuras labiopalatinas de crianças nascidas na cidade de Joinville/SC no período de 1994 a 2000 [dissertação]. Joinville: Universidade Federal de Santa Catarina, Faculdade de Odontologia; 2002.
27. Oliveira RP. Relação entre os sinais clínicos da fissura de palato submucosa e a sintomatologia especifica: uma abordagem preventiva [dissertação]. Bauru: Universidade de São Paulo, Hospital de Reabilitação de Anomalias Craniofaciais; 2002.

28. Miguel HC, Genaro KF, Trindade IE. Avaliação perceptiva e instrumental da função velofaríngea na fissura de palato submucosa assintomática. Pró-Fono. 2007;19(1):105-12.

29. Jesus MS, Lima RC, Valente P, Silva EB, Matos EF, Di Ninno CQ. Características de um grupo de pacientes com fissura labiopalatina atendidos na clínica de fonoaudiologia do Centrare - PUC-Minas/ Hospital da Baleia. Rev Cient Inst Metodista Izabela Hendrix [Internet]. 2008. Disponível em: http://proacad.metodistademinas.edu.br/tecer/ TEXTOS_TECER0/IMGS/PDF/CARACTERISTICAS_MARISA.pdf

30. Silva EB, Furia CL, Di Ninno CQ. Aleitamento materno em recém nascidos portadores de fissura labiopalatina: dificuldades e métodos utilizados. Rev CEFAC. 2008;7(1):21-8.

Anexo 1. Protocolo de coleta de dados em pacientes com fissura labial

Iniciais:

DN: 11 _
Prontuário:

Data:

Fissura pré-forame: ( ) unilateral direita ( ) unilateral esquerda ( ) bilateral
( ) completa
( ) incompleta
Palato:
( ) íntegro ( ) chanfradura óssea ( ) diástase muscular ( ) úvula bífida

( ) úvula sulcada ( ) zona translúcida

Sintomas: ( ) aleitamento materno até __ meses; ( ) refluxo nasal de alimentos

( ) _ episódios de otite

Avaliação da fala em:

( ) fala normal ( ) Hipernasalidade () DAC ( ) EAN () Fp 